\title{
ESTIMATIVAS DE PARÂMETROS GENÉTICOS E GANHOS DE SELEÇÃO EM PROGÊNIES DE POLINIZAÇÃO ABERTA DE AÇAIZEIRO ${ }^{1}$
}

\author{
JOÃOTOMÉ DE FARIAS NETO², MARCOS DEON VILELA DE RESENDE ${ }^{3}$, \\ MARIA DO SOCORRO PADILHA DE OLIVEIRA ${ }^{2}$, OSCAR LAMEIRANOGUEIRA ${ }^{2}$, \\ PETER NILTON BEZERRA FALCÃO ${ }^{4}$, NELMA SANTOS AMORIM DOS SANTOS ${ }^{4}$
}

RESUMO - O presente trabalho teve como objetivos estudar a variabilidade genética, estimar parâmetros genéticos e fenotípicos, e realizar a predição de valores genéticos dos indivíduos de açaizeiro irrigado no Estado do Pará, utilizando a metodologia BLUP/REML a partir da avaliação de progênies com base nos caracteres altura do primeiro cacho (APC), peso total do cacho (PTC), peso total de frutos $(\mathrm{PTF})$, número de cachos (NC), peso médio do cacho (PMC), comprimento médio da ráquis (CMR), número médio de ráquilas (NMR), número de perfilhos (NP) e peso médio de cem frutos (P100). Cinqüenta progênies foram avaliadas em dois látices $5 \times 5$ com duas repetições e parcelas lineares de cinco plantas cada, no espaçamento de $5 \mathrm{~m} \mathrm{x} 5 \mathrm{~m}$. O programa computacional Selegen- Reml/ Blup foi utilizado para as análises genéticas e a identificação dos melhores indivíduos para compor a população de produção de sementes para um programa a curto prazo e a de melhoramento para um programa a longo prazo. As correlações genotípicas de maiores magnitudes foram aquelas envolvendo o peso total de frutos e peso total do cacho, peso total do cacho e número de cacho e peso total de fruto e número de cacho, indicando que a seleção para peso total de frutos pode ser realizada por meio da seleção daquela de mais fácil seleção. As estimativas dos parâmetros genéticos obtidos revelam excelente potencial seletivo da população e variabilidade genética suficiente para o melhoramento genético da população a curto e longo prazos. Ganho genético considerável de 45,33\% em relação à média do experimento pode ser obtido com a seleção dos 20 melhores indivíduos para o caráter produção total de frutos. Termos para indexação: predição de ganhos, parâmetros genéticos, Reml/Blup, frutífera.

\section{ESTIMATES OF GENETIC PARAMETERS AND SELECTION GAINS IN PROGENYES OF OPEN POLLINATION OF AÇAI TREE (Euterpe oleracea)}

ABSTRACT- The present work aimed to study the genetic variability, estimate the genetic and phenotypic parameters and to conduct the prediction of genetic values of individuals of irrigated açai tree in the state of Pará. The objective was the selection and genetic improvement of the production of fruits, using the REML/BLUP procedure applied in the progenies for the characters height of the first cluster, total cluster mass, total fruit mass, number of clusters, mean cluster weight, average length of the leaf rachis, average number of rachillas, number of tillers and average mass of one hundred fruits. Fifty progenies were evaluated in two $5 \times 5$ lattice design with two replications and linear plots- of five plants each, in a $5 \mathrm{~m}$ x $5 \mathrm{~m}$ spacing. The software Selegen- Reml/Blup was used for the genetic analyses and identification of the best individuals to compose the population for seed production (short-term program) and of improvement (long-term program). The estimation of genotipic correlation was high between total fruit mass and number of cluster and average among total fruit mass and average cluster mass and number of rachillas. The estimates of the genetic parameters obtained in the present study show excellent selective potential of the population and sufficient genetic variability for the genetic improvement of the population in short and long terms. Considerable genetic gain of $45.33 \%$ relative to the average of the experiment can be obtained with the election of the 20 best individuals for the character total production of fruits.

Index terms: predicting gain, genetic parameters, Reml/Blup, Euterpe oleracea, fruit tree.

\section{INTRODUÇÃO}

O açaizeiro (Euterpe oleracea Mart.) é uma espécie frutífera e produtora de palmito, nativa da Amazônia e tem com centro de origem o Estado do Pará, que é o maior produtor e o principal consumidor, onde estão estabelecidas densas e diversificadas populações naturais.

Atualmente, a demanda e o potencial de mercado do açaí cresceram de maneira significativa e deixaram de possuir uma dimensão regional para ganhar importância nacional e, mais recentemente, internacional, graças à qualidade nutricional da bebida resultante da maceração dos seus frutos, denominada vinho ou suco de açai. $\mathrm{O}$ crescimento do mercado do açaizeiro está associado aos benefícios à saúde que a ciência vem atribuindo à ingestão desse alimento que apresenta baixo nível de calorias, altas concentrações de vitaminas, fibras, sais minerais e elevado teor de antocianinas (Rogez, 2000).

Trata-se de uma espécie perene, que é propagada principalmente por sementes (Jardim, 1991; Oliveira et al., 2002), preferencialmente alógama, com 12,9\% de autofecundação em condições naturais (Souza, 2002), tendo como principal

1(Trabalho 003-08). Recebido em: 02-01-2008. Aceito para publicação em: 26-05-2008.

${ }^{2}$ Pesquisadores da Embrapa Amazônia Oriental, Trav. Dr. Enéas Pinheiro, s/n Caixa Postal 48, 66095-100 Belém-PA. tome@cpatu.embrapa.br, spadilha@cpatu.embrapa.br, oscar@cpatu.embrapa.br

${ }_{3}^{3}$ Pesquisador da Embrapa Florestas, Estrada da Ribeira, km 11, Caixa Postal 319, 83411-000 Colombo-PR.

Rev. Bras. Frutic., Jaboticabal - SP, v. 30, n. 4, p. 1051-1056, Dezembro 2008 
característica a formação de agrupamentos de estipes, denominados touceira (Calzavara, 1988), e para Clement (1992) trata-se de uma espécie semidomesticada ou em fase de domesticação.

O açaizeiro encontra condições ideais de cultivo nas faixas climáticas com regular distribuição de chuvas e em áreas que, mesmo com período de estiagem definido, disponham de umidade satisfatória no solo, como nas várzeas. Quando cultivado em área de terra firme, com tipo climático Ami (apresenta índice pluviométrico anual que define uma estação relativamente de estiagem, mas com precipitação acima de $2.500 \mathrm{~mm}$ anual) e Awi (índice pluviométrico anual entre 1.000 e 2.500 mm, com nítida estação de estiagem), para evitar a redução ou paralisação do crescimento, floração e frutificação, é importante planejar, nos períodos menos chuvosos, a utilização de irrigação (Calzavara, 1972). Para Taiz \& Zeiger (2004), a resposta ao estresse hídrico limita o tamanho e o número de folhas, levando à redução do consumo de carbono e energia por esse órgão da planta, ocorrendo grande alocação de fotoassimilados para o sistema radicular. Observa-se, também, redução da expansão foliar, altura e diâmetro das plantas, resultando em perda da biomassa vegetal.

O programa de melhoramento genético do açaizeiro para as condições de terra firme irrigado na Embrapa Amazônia Oriental é recente, e o método de seleção empregado tem sido os testes de progênies de polinização livre, que são úteis para os objetivos de seleção, melhoria genética e o estudo dos parâmetros genéticos. Estudos realizados por Farias Neto et al. (2005, 2007), empregando características vegetativas em açaizeiro, sugerem a existência de variabilidade entre e dentro de procedência/ progênies. Entretanto, estimativas de parâmetros genéticos envolvendo características produtivas inexistem na literatura.

Em espécies perenes, a etapa de avaliação é a mais onerosa e demorada nos programas de melhoramento genético. Assim, a aplicação de metodologias eficientes que aumentem a acurácia no processo seletivo, é de extrema importância.

Segundo Resende \& Dias (2000), no melhoramento de espécies perenes, o uso de técnicas de avaliação genética, com base em modelos mistos tipo REML/BLUP (máxima verossimilhança restrita/melhor predição linear não-viciada), é fundamental para a predição de valores genéticos aditivos e genotípicos de indivíduos com potencial para a seleção, a nível intrapopulacional e interpopulacional. O procedimento REML/ BLUP tem sido usado no melhoramento de palmáceas para a seleção de progênies e indivíduos em pupunheira (Farias Neto $\&$ Resende, 2001) e palmeira-real (Bovi et al. 2003; 2004).

O presente trabalho teve como objetivos estudar a variabilidade genética, estimar parâmetros genéticos e fenotípicos e realizar a predição de valores genéticos dos indivíduos de açaizeiro irrigado no Estado do Pará, visando à seleção e melhoramento genético da produção de frutos, empregando a metodologia REML/BLUP.

\section{MATERIAL E MÉTODOS}

O estudo foi realizado na base experimental da Embrapa Amazônia Oriental, localizada no município de Tomé-Açu, no nordeste paraense, aproximadamente entre as latitudes de $01^{\circ} 5738^{\prime \prime}$ e $03^{\circ} 16^{\prime} 37^{\prime}$ " S e as longitudes de $47^{\circ} 53^{\prime} 32^{\prime \prime} \mathrm{W}$ e 48 49'15W. Os parâmetros meteorológicos climáticos indicam clima quente e úmido, ajustando-se ao tipo climático Ami, da classificação de Köppen (Pacheco \& Bastos, 2001). A precipitação pluviométrica é caracterizada por dois períodos distintos de chuvas, um de dezembro a maio, com índices superiores a $150 \mathrm{~mm} / \mathrm{mês}$, onde se concentram cerca de $80 \%$ do total anual de precipitação, e outro, de junho a novembro, com índice variando de $49 \mathrm{~mm}$ a $105 \mathrm{~mm}$.

Os experimentos foram estabelecidos em março de 2003 em 2 látices 5 x 5 com duas repetições e 5 plantas por parcela, no espaçamento de $5 \mathrm{~m} \times 5 \mathrm{~m}$, totalizando 50 progênies, além de uma bordadura externa ao experimento. Os tratamentos foram representados por progênies de polinização livre, originadas de plantas selecionadas nos municípios de Afuá e Chaves, região norte da Ilha de Marajó. Essa região apresenta como principal característica a produção de frutos em período considerado como entressafra na região próxima à cidade de Belém.

Na seleção fenotípica das árvores em condições naturais, utilizaram-se como critérios de seleção o número de cacho por planta, o tamanho do cacho, a presença de perfilhos e o estado fitossanitário das plantas.

A avaliação das progênies teve início em agosto de 2006, quando as plantas estavam com 3,5 anos até setembro de 2007 (4,5 anos) ao nível de indivíduos para os caracteres altura do primeiro cacho (APC), peso total do cacho (PTC), peso total de fruto (PTF), número de cacho (NC), peso médio do cacho (PMC), comprimento médio da ráquis $(\mathrm{CMR})$, número médio de ráquilas (NMR), número de perfilhos (NP) e peso médio de cem frutos (PM100). Os dados encontram-se desbalanceados em virtude da perda (morte) de algumas plantas nas parcelas. Atualmente, a colheita dos frutos é realizada quinzenalmente.

Para a análise dos dados, foi empregada a metodologia de modelos lineares mistos (procedimento REML/BLUP) para o delineamento de blocos incompletos desbalanceado. Isto permitiu o ajuste para os gradientes ambientais de blocos e também a recuperação de informação genética interblocos incompletos, propiciando eficiente estimação de parâmetros e seleção em nível de indivíduos. Os efeitos de blocos foram considerados como aleatórios, visando a propiciar a recuperação de informação genética interblocos. A metodologia REML/BLUP produz, simultaneamente, três tipos de informação: estimativas dos efeitos genéticos (e conseqüentemente do ganho ou progressão genética), estimativas dos efeitos ambientais e estimativas dos componentes de variância genética e ambiental (parâmetros genéticos).

Os parâmetros genéticos foram estimados via REML, e os genéticos aditivos individuais foram estimados pelo procedimento BLUP, por meio do software genético-estatístico Selegen-Reml/Blup (Resende, 2002).

Utilizou-se do seguinte modelo estatístico para a avaliação genética (Resende, 2002):

$\mathrm{y}=\mathrm{Xm}+\mathrm{Za}+\mathrm{Wp}+\mathrm{Tb}+\mathrm{e}$, em que:

$\mathrm{y}, \mathrm{m}, \mathrm{a}, \mathrm{p}, \mathrm{b}, \mathrm{e}=$ vetores de dados, de efeitos da média geral (fixo), de efeitos genéticos aditivos individuais (aleatórios), 
de efeitos de parcela (aleatórios), de efeitos de blocos (aleatórios) e de erros aleatórios, respectivamente.

$\mathrm{X}, \mathrm{Z}, \mathrm{W}, \mathrm{T}=$ matrizes de incidência para $\mathrm{m}, \mathrm{a}, \mathrm{p}$ e $\mathrm{b}$, respectivamente.

Associados a esse modelo, têm-se as seguintes distribuições e estruturas de médias e variâncias:

$$
\begin{aligned}
& y \mid m, V \sim N(X m, V) \\
& a \mid A, \sigma_{a}^{2} \sim N\left(0, \quad A \sigma_{a}^{2}\right) \\
& c \mid \sigma_{c}^{2} \sim N\left(0, I \sigma_{c}^{2}\right) \\
& b \mid \sigma_{b}^{2} \sim N\left(0, I \sigma_{b}^{2}\right) \\
& e \mid \sigma_{e}^{2} \sim N\left(0, I \sigma_{e}^{2}\right)
\end{aligned}
$$

As co-variâncias entre todos os efeitos aleatórios do modelo são consideradas nulas.

Assim:

$E\left[\begin{array}{l}y \\ a \\ c \\ b \\ e\end{array}\right]=\left[\begin{array}{c}X m \\ 0 \\ 0 \\ 0 \\ 0\end{array}\right]$ e Var $\left[\begin{array}{c}y \\ a \\ c \\ b \\ e\end{array}\right]=\left[\begin{array}{ccccc}V & Z G & W C & T B & R \\ G Z & G & 0 & 0 & 0 \\ C W & 0 & C & 0 & 0 \\ B T & 0 & 0 & B & 0 \\ R & 0 & 0 & 0 & R\end{array}\right]$ em que:

$G=A \sigma_{A}^{2}$

$C=I \sigma_{c}^{2}$

$B=I \sigma_{b}^{2}$

$R=I \sigma_{e}^{2}$

$V=Z A \sigma_{a}^{2} Z^{\prime}+W I \sigma_{c}^{2} W^{\prime}+T^{\prime} I \sigma_{\hat{\theta}}^{2} T+I \sigma_{e}^{2}=Z G Z^{\prime}+W C W^{\prime}+T B T^{\prime}+R$.

As equações de modelo misto para a predição BLUP dos valores genéticos individuais equivalem a:

$$
\left[\begin{array}{cccc}
X^{\prime} X & X^{\prime} Z & X^{\prime} W & X^{\prime} T \\
Z^{\prime} X & Z^{\prime} Z+A^{-1} \lambda_{1} & Z^{\prime} W & Z^{\prime} T \\
W^{\prime} X & W^{\prime} Z & W^{\prime} W+\lambda_{2} & W^{\prime} T \\
T^{\prime} X & T^{\prime} Z & T^{\prime} W & T^{\prime} T+I \lambda_{3}
\end{array}\right]\left[\begin{array}{c}
\hat{b} \\
\hat{a} \\
\hat{c} \\
\hat{b}
\end{array}\right]=\left[\begin{array}{c}
X^{\prime} y \\
Z^{\prime} y \\
W^{\prime} y \\
T^{\prime} y
\end{array}\right] \text { em que }
$$

$\lambda_{1}=\frac{\sigma_{e}^{2}}{\sigma_{a}^{2}}=\frac{1-h^{2}-c^{2}-b^{2}}{h^{2}} ; \lambda_{2}=\frac{\sigma_{e}^{2}}{\sigma_{c}^{2}}=\frac{1-h^{2}-c^{2}-b^{2}}{c^{2}} \quad \lambda_{3}=\frac{\sigma_{e}^{2}}{\sigma_{\partial}^{2}}=\frac{1-h^{2}-c^{2}-b^{2}}{b^{2}}$ $h^{2}=\frac{\sigma_{a}^{2}}{\sigma_{a}^{2}+\sigma_{c}^{2}+\sigma_{e}^{2}+\sigma_{b}^{2}} \quad:$ herdabilidade individual no sentido restrito.

$c^{2}=\sigma_{c}^{2} /\left(\sigma_{a}^{2}+\sigma_{c}^{2}+\sigma_{e}^{2}+\sigma_{b}^{2}\right) \quad:$ correlação devida ao ambiente comum da parcela.

$b^{2}=\sigma_{\partial}^{2} /\left(\sigma_{a}^{2}+\sigma_{c}^{2}+\sigma_{e}^{2}+\sigma_{b}^{2}\right) \quad$ : correlação devida ao ambiente comum do bloco dentro de repetição. $\sigma_{\mathrm{a}}^{2}$ : variância genética aditiva.

$\sigma_{\mathrm{c}}^{2}$ : variância entre parcelas.

$\sigma_{b}^{2}$ : variância entre blocos.

$\sigma_{\mathrm{e}}^{2}$ : variância residual (ambiental dentro de parcelas + não aditiva).

A: matriz de correlação genética aditiva entre os indivíduos em avaliação.

Os estimadores iterativos dos componentes de variância por REML via algoritmo EM equivalem a:

$\hat{\sigma}_{e}^{2}=\left[\begin{array}{llll}y^{\prime} y-\hat{b}^{\prime} & X^{\prime} y-\hat{a}^{\prime} \quad Z^{\prime} y-\hat{c}^{\prime} \quad W^{\prime} y-\hat{b}^{\prime} T^{\prime} y\end{array}\right] /[N-r(x)]$

$\hat{\sigma}_{a}^{2}=\left[\hat{a}^{\prime} A^{-1} \hat{a}+\hat{\sigma}_{e}^{2} \operatorname{tr}\left(A^{-1} C^{22}\right)\right] / q$

$\hat{\sigma}_{c}^{2}=\left[\hat{c}^{\prime} c+\hat{\sigma}_{e}^{2} \operatorname{tr} C^{33}\right] / s$, em que:

$\hat{\sigma}_{b}^{2}=\left[\hat{b}^{\prime} \hat{b}+\hat{\sigma}_{e}^{2} \operatorname{tr} C^{44}\right] / \eta$, em que:

$C^{22}, C^{33} e C^{44}$ advêm da inversa da matriz dos coeficientes das equações de modelo misto.

tr: operador traço matricial.

$\mathrm{r}(\mathrm{x})$ : posto da matriz $X$.

$\mathrm{N}, \mathrm{q}, \mathrm{s}, \eta$ : número total de dados, de indivíduos, de parcelas e de blocos, respectivamente.

Com base nos valores genéticos individuais, os ganhos genéticos esperados com a prática da seleção para peso total de frutos foram estimados admitindo-se duas estratégias de seleção: 20 indivíduos pertencentes a 9 progênies, representando a população de produção de sementes, simulando o programa a curto prazo e com 50 indivíduos, pertencentes a 23 progênies, para compor a população de melhoramento, simulando o programa a longo prazo.

\section{RESULTADOS E DISCUSSÃO}

Nas Tabelas 1 e 2, são apresentados os componentes de variância e as estimativas dos parâmetros genéticos e fenotípicos associados aos nove caracteres. Constata-se ampla variabilidade genética para os caracteres APC, PMC e NMR, conduzindo a altos valores de herdabilidade a nível individual e média de progênies. Os valores moderados de herdabilidade foram estimados para os caracteres PTC e PTF.

Esses fatos associados a componentes de variância genética relativa aos caracteres revelam excelentes possibilidades de seleção na população experimental. Apesar de os caracteres CMR e NC terem apresentado coeficientes de herdabilidade de baixa magnitude em nível de indivíduo de 10,7675\% e 13,4008\%, existem boas possibilidades de seleção, pois conduziram as estimativas de herdabilidade de média de progênies igual a $35,3023 \%$ e $36,2814 \%$ e acurácias na seleção de progênies de $59,4157 \%$ e $60,2340 \%$, respectivamente. Em geral, herdabilidades individuais de baixa magnitude são comuns para caracteres quantitativos e, via de regra, conduzem a moderadas magnitudes das herdabilidades em nível de médias de progênies (Resende, 2002).

Os coeficientes de variação experimental $\left(\mathrm{CV}_{\mathrm{e}} \%\right)$ obtidos para os caracteres APC, PMC, CMR, NMR, P100 e NP podem ser 
considerados baixos e demonstram boa precisão. Já para os caracteres PTC e PTF, estes coeficientes foram 34,1253\% e $36,1971 \%$, respectivamente, valores altos, indicando grande variação dos dados.

Os coeficientes de determinação dos efeitos de parcela $\left(c^{2}\right)$ ficaram entre $4,048 \%$ e $9,133 \%$ para todos os caracteres, exceção para os caracteres NMR, CMR e P100, que apresentaram valores próximos de zero, revelando que pequena variação ambiental permaneceu dentro das parcelas.

Os valores de $\mathrm{c}^{2}$ observados em bons experimentos, em plantas perenes, situam-se em torno de $10 \%$, quando a herdabilidade estimada é da ordem de $30 \%$, ou seja, $10 \%$ da variação fenotípica total dentro do bloco. Assim, para o nível de $30 \%$ de herdabilidade individual, $\mathrm{c}^{\wedge} 2>0,10$ pode ser classificado como baixo e $\mathrm{c}^{\wedge} 2>0,10$ pode ser classificado como alto, permitindo, assim, inferência sobre a variabilidade espacial dentro dos blocos.

Valores altos de $\mathrm{c}^{2}$ indicam alta variabilidade entre parcelas dentro de blocos e alta correlação ambiental entre observações dentro de parcelas (Sturion \& Resende, 2004). No presente estudo, mesmo para aquelas herdabilidades de menores magnitudes, como para PTF, cuja herdabilidade foi igual a $29,4831 \%$, o c é é considerado baixo.

As estimativas do coeficiente de determinação dos efeitos de bloco foram baixas, com exceção do caráter APC $(13,2541 \%)$, indicando que o delineamento em látice foi importante para aqueles caracteres. Os ajustes para todas essas variações entre parcelas e entre blocos foram realizados pelo procedimento REML/BLUP, que forneceu os valores genéticos livres de todos esses efeitos.

As estimativas de correlações genotípicas entre os nove caracteres, que refletem associações de natureza herdável, são apresentadas na Tabela 3. As correlações genotípicas de maiores magnitudes foram aquelas envolvendo peso total do cacho e peso total de frutos $(0,9916)$, peso total do cacho e número de cacho $(0,8941)$ e peso total de fruto e número de cacho $(0,8864)$, indicando que é possível aumentar o peso total de frutos por intermédio de seleção indireta para peso total de cachos e número de cachos. O peso total de frutos apresentou valores de média magnitude com número médio de ráquilas $(0,4922)$ e com peso médio do cacho $(0,4456)$. Quanto à correlação entre o peso de cem frutos e produção total de frutos, a estimativa foi baixa $(0,2659)$, indicando ser possível a obtenção de progênies produtivas apresentando menor peso ou menor tamanho do fruto. Esse fato é de máxima importância, haja vista que os processadores de açaí preferem tamanho de fruto pequeno.

Os resultados referentes à seleção de indivíduos com base em seus valores genéticos aditivos são apresentados na Tabela 4. A determinação do número adequado de indivíduos a serem selecionados deve considerar a endogamia na geração de plantio, para a composição da população de produção de sementes e a manutenção de tamanho efetivo populacional compatível com a obtenção do limite seletivo para a composição da população de melhoramento (Resende \& Bertolucci, 1995). No presente trabalho, maximizou-se o ganho, preestabelecendo um tamanho efetivo mínimo de 12,43 e 29,29 para a composição das populações de produção de sementes e de melhoramento, respectivamente Verificam-se altos ganhos genéticos empregando as duas modalidades de seleção. A seleção dos 20 indivíduos visando a transformar o teste em pomar de sementes por muda, simulando um programa a curto prazo, é representado por 9 famílias e propicia ganhos estimado de $45,33 \%$ em relação à média, fato que eleva a média do caráter PTF de $8.966,81 \mathrm{~kg}$ para $13.031,72 \mathrm{~kg}$, com tamanho efetivo populacional de 12,43, suficiente para prevenir a ocorrência de depressão endogâmica na geração de plantio.

Por outro lado, a seleção dos 50 melhores indivíduos correspondente a, aproximadamente, $10 \%$ do total dos indivíduos existentes no experimento para o estabelecimento de uma população de melhoramento, simulando programa a longo prazo, é constituída por 23 progênies e proporcionou um ganho estimado de $28,09 \%$ em relação à média, elevando a média populacional de $8.966,81 \mathrm{~kg}$ para $11.486,19 \mathrm{~kg}$ e um tamanho efetivo de 29,29, suficiente para a manutenção da variabilidade genética e a obtenção de ganhos em ciclos subseqüentes de seleção. Vale ressaltar que os valores genéticos aditivos são também úteis no planejamento dos cruzamentos para avaliação no próximo ciclo seletivo, podendo os indivíduos com os maiores valores genéticos aditivos participarem de maior número de cruzamentos.

TABELA 1 - Estimativas dos componentes de variância e parâmetros genéticos e fenotípicos para os caracteres altura médio do primeiro cacho (APC), peso total do cacho (PTC), peso total de frutos (PTF) e número de cachos (NC) em progênies de açaizeiro irrigado. Belém-PA. 2007.

\begin{tabular}{lcccc}
\hline \multicolumn{1}{c}{ Parâmetro } & APC & PTC & PTF & NC \\
\hline Variância genética aditiva individual & 0,0831 & 24889583,781 & 12784534,868 & 1,8182 \\
Variância entre parcelas & 0,0112 & 5933410,630 & 3187853,972 & 0,7596 \\
Variância entre blocos & 0,0163 & 385829,138 & 243900,069 & 0,1857 \\
Variância residual & 0,0127 & 49644871,574 & 27146007,666 & 10,8045 \\
Variância fenotípica & 0,1234 & 80853695,124 & 43362296,577 & 13,5681 \\
Herdabilidade aditiva individual (\%) & 67,347 & 30,7835 & 29,4831 & 13,4008 \\
$\begin{array}{l}\text { Coeficiente de determinação de parcelas (\%) } \\
\text { Coeficiente de determinação de blocos (\%) }\end{array}$ & 9,1332 & 7,3385 & 7,3517 & 5,5984 \\
$\begin{array}{l}\text { Coeficiente de variação genética aditiva } \\
\text { individual (\%) }\end{array}$ & 13,2541 & 0,4772 & 0,5625 & 1,3691 \\
$\begin{array}{l}\text { Coeficiente de variação genotípica progênie } \\
\text { (\%) }\end{array}$ & 13,7321 & 38,4595 & 39,8753 & 21,2133 \\
Coeficiente de variação experimental (\%) & 6,8660 & 19,2297 & 19,9376 & 10,6066 \\
Herdabilidade da média de progênie ajustada & 7,7211 & 34,1253 & 36,1971 & 28,1125 \\
(\%) & 75,9821 & 55,9500 & 54,8239 & 36,2814 \\
Herdabilidade dentro de progênie (\%) & 83,1088 & 27,3263 & 26,1020 & 11,2069 \\
Acurácia na seleção de progênie & 87,1677 & 74,7997 & 74,0432 & 60,2340 \\
\hline Média Geral & 2,0991 & 12971,9349 & 8966,8111 & 6,3564 \\
\hline
\end{tabular}

Rev. Bras. Frutic., Jaboticabal - SP, v. 30, n. 4, p.1051-1056, Dezembro 2008 
TABELA 2 - Estimativas dos componentes de variância e parâmetros genéticos e fenotípicos para os caracteres peso médio do cacho (PMC), comprimento médio da ráquis (CMR), número médio de ráquilas (NMR), número de perfilhos (NP) e peso de cem frutos (P100) em açaizeiro sob irrigação. Belém-PA. 2007.

\begin{tabular}{lccccc}
\hline \multicolumn{1}{c}{ Parâmetro } & PMC & CMR & NMR & NP & P100 \\
\hline Variância genética aditiva individual & 21728,425 & 10,248 & 109,3706 & 0,5359 & 705,7515 \\
Variância entre parcelas & 1651,972 & 0,427 & 0,2463 & 0,05069 & 2,4008 \\
Variância entre blocos & 2062,695 & 0,415 & 0,7618 & 0,0011 & 0,2197 \\
Variância residual & 5334,979 & 84,085 & 31,3221 & 0,6644 & $-260,539$ \\
Variância fenotípica & 30778,070 & 95,1751 & 141,7008 & 1,2522 & 447,8331 \\
Herdabilidade aditiva individual (\%) & 70,5971 & 10,7675 & 77,1842 & 42,7983 & - \\
Coeficiente de determinação de parcelas (\%) & 5,3674 & 0,4487 & 0,1738 & 4,0483 & 0,5361 \\
Coeficiente de determinação de blocos (\%) & 6,7018 & 0,4363 & 0,5376 & 0,0902 & 0,0491 \\
Coeficiente de variação genética aditiva & 23,6259 & 6,0614 & 11,5447 & 22,9794 & 20,7166 \\
individual (\%) & & & & & \\
Coeficiente de variação genotípica progênie & 11,8129 & 3,0307 & 5,7723 & 11,4897 & 10,3583 \\
(\%) & & & & \\
Coeficiente de variação experimental (\%) & 12,3935 & 8,2057 & 5,2845 & 16,1275 & 5,8437 \\
Herdabilidade da média de progênie ajustada & 78,4231 & 35,3023 & 82,6768 & 66,9990 & 92,6296 \\
(\%) & & & & \\
Herdabilidade dentro de progênie (\%) & 75,3368 & 8,3752 & 72,3669 & 37,6914 & - \\
Acurácia na seleção de progênie & 88,5568 & 59,4157 & 90,9268 & 81,8529 & 96,2443 \\
\hline Média Geral & 623,9145 & 52,8136 & 90,5867 & 3,1858 & 128,2349 \\
\hline
\end{tabular}

TABELA 3 - Estimativas de correlações genotípicas entre os caracteres altura do primeiro cacho (APC), peso total do cacho (PTC), peso total de frutos (PTF), peso médio do cacho (PMC), comprimento médio dos ráquis (CMR), número médio de ráquilas (NMR), peso médio de cem sementes (P100), número de perfilhos (NP) e número de cachos (NC) em açaizeiro. Belém-PA. 2007.

\begin{tabular}{|c|c|c|c|c|c|c|c|c|c|}
\hline Variável & APC & PTC & PTF & PMC & CMR & NMR & P100 & NP & $\mathrm{NC}$ \\
\hline APC & 1 & $-0,4120$ & $-0,4118$ & $-0,2984$ & $-0,0311$ & $-0,1814$ & $-0,1108$ & 0,1497 & $-0,3506$ \\
\hline PTC & & 1 & 0,9916 & 0,5130 & 0,1688 & 0,5252 & 0,2803 & $-0,2373$ & 0,8941 \\
\hline PTF & & & 1 & 0,4456 & 0,0917 & 0,4922 & 0,2659 & $-0,2386$ & 0,8864 \\
\hline PMC & & & & 1 & 0,4859 & 0,6238 & 0,4334 & $-0,1962$ & 0,1916 \\
\hline CMR & & & & & 1 & 0,2470 & 0,2299 & 0,2496 & 0,0506 \\
\hline NMR & & & & & & 1 & 0,1212 & $-0,2371$ & 0,2682 \\
\hline P100 & & & & & & & 1 & 0,0372 & 0,1469 \\
\hline NP & & & & & & & & 1 & $-0,1628$ \\
\hline $\mathrm{NC}$ & & & & & & & & & 1 \\
\hline
\end{tabular}

TABELA 4 - Valores genéticos aditivos individuais, ganho genético e tamanho efetivo populacional (Ne) com a seleção dos 20 melhores indivíduos e do indivíduo de número 50 para o caráter produção total de frutos, em açaizeiro, visando à propagação sexuada. Belém-PA. 2007.

\begin{tabular}{|c|c|c|c|c|c|c|}
\hline Ordem & Família & Planta & $\begin{array}{c}\text { Valor } \\
\text { Fenotípico }\end{array}$ & $\begin{array}{c}\text { Valor Genético } \\
\text { Aditivo }\end{array}$ & $\begin{array}{l}\text { Ganho Genético } \\
\text { Acumulado (\%) }\end{array}$ & $\mathrm{Ne}$ \\
\hline 1 & 04 & 3 & 35366 & 16760,19 & 86,91 & 1,00 \\
\hline 2 & 11 & 2 & 30108 & 16145,22 & 80,05 & 2,00 \\
\hline 3 & 37 & 5 & 30677 & 15444,58 & 72,24 & 3,00 \\
\hline 4 & 11 & 2 & 25810 & 15228,28 & 69,83 & 3,49 \\
\hline 5 & 26 & 1 & 29930 & 15063,84 & 68,00 & 4,49 \\
\hline 6 & 11 & 1 & 23272 & 14360,89 & 60,16 & 4,64 \\
\hline 7 & 11 & 4 & 22384 & 14129,11 & 57,57 & 4,64 \\
\hline 8 & 20 & 3 & 23988 & 14033,13 & 56,50 & 5,59 \\
\hline 9 & 11 & 3 & 21240 & 13830,50 & 54,24 & 5,47 \\
\hline 10 & 47 & 1 & 25548 & 13804,41 & 53,95 & 6,38 \\
\hline 11 & 47 & 4 & 22892 & 13698,18 & 52,79 & 7,05 \\
\hline 12 & 31 & 3 & 26396 & 13484,55 & 50,38 & 7,97 \\
\hline 13 & 31 & 1 & 26244 & 13444,88 & 49,94 & 8,65 \\
\hline 14 & 21 & 5 & 27224 & 13347,49 & 48,85 & 9,56 \\
\hline 15 & 50 & 1 & 24564 & 13334,14 & 48,71 & 10,49 \\
\hline 16 & 04 & 5 & 22192 & 13321,52 & 48,56 & 11,16 \\
\hline 17 & 20 & 4 & 20798 & 13301,61 & 48,34 & 11,84 \\
\hline 18 & 20 & 1 & 20712 & 13178,03 & 46,96 & 12,25 \\
\hline 19 & 20 & 2 & 20458 & 13111,73 & 46.22 & 12,43 \\
\hline 20 & 20 & 2 & 19764 & 13031,72 & 45,33 & 12,43 \\
\hline $\mathrm{n}$ & $\mathrm{n}$ & $\mathrm{n}$ & $\mathrm{n}$ & $\mathrm{n}$ & $\mathrm{n}$ & $\mathrm{n}$ \\
\hline 50 & 26 & 4 & 13112 & 11486,1980 & 28,09 & 29,29 \\
\hline
\end{tabular}




\section{CONCLUSÕES}

1-As correlações genotípicas de maiores magnitudes foram aquelas envolvendo o peso total de frutos e o peso total do cacho, peso total do cacho e número de cacho, e peso total de fruto e número de cacho, indicando que a seleção para peso total de frutos pode ser realizada por meio da seleção daquela de mais fácil seleção.

2-As estimativas dos parâmetros genéticos obtidos revelam excelente potencial seletivo da população e variabilidade genética suficiente para o melhoramento genético da população a curto e longo prazos.

3-Ganho genético considerável de 45,33\% em relação à média do experimento pode ser obtido com a seleção dos 20 melhores indivíduos para o caráter produção total de frutos.

\section{AGRADECIMENTOS}

À Secretária de Ciência e Tecnologia do Estado do Pará (SECTAM/FUNTEC), ao Conselho Nacional de Desenvolvimento Científico e Tecnológico (CNPq) e à Agência de Desenvolvimento da Amazônia (ADA), pelo apoio financeiro.

\section{REFERÊNCIAS}

BOVI, M.L.A.; RESENDE, M.D.V.; SPIERING, S.H. Genetic parameters estimation in King palm through a mixed mating system model. Horticultura Brasileira, Brasília, v. 21, n. 1, p. 93-98, 2003.

BOVI, M.L.A.; RESENDE, M.D.V.; SÁES, L.A.; UZZO, R.P. Genetic analysis for sooty mold resistance and heart of palm yield in Archontophoenix. Scientia Agricola, Piracicaba, v, 61, n, 2, p, 178-184, 2004.

CALZAVARA, B.B.G. As possibilidades do açaizeiro no estuário Amazônico. Belém-PA: FCAP, 1972. 103p. (Boletim, 5).

CALZAVARA, B.B.G. Importância do açaizeiro (Euterpe oleracea Mart.) como produtor de frutos e palmito para o Estado do Pará. In: ENCONTRO NACIONAL DE PESQUISADORES EM PALMITO, 1., 1987. Curitiba. Anais... Curitiba: Embrapa-CNPF, 1988. p. 249-259.

CLEMENT, C.R. Domesticated palms. Principes, Lawrence, v. 36, n.2, p. 70-78. 1992.

FARIAS NETO, J.T. de; RESENDE, M.D.V. Aplicação da metodologia de modelos mistos (REML/BLUP) na estimação de componentes de variância e predição de valores genéticos em pupunheira (Bactris gasipaes). Revista Brasileira de Fruticultura, Jaboticabal, v. 23, n. 2, p. 320-324, 2001.

FARIAS NETO, J.T. de; OLIVEIRA, M.S.P. de; MULLER, A.A.; NOGUEIRA, O.L.; ANAISSI, D.F.S.P. Variabilidade genética em progênies jovens de açaizeiro. Cerne, Lavras, v.11, n.4, p.336341, 2005.
FARIAS NETO, J.T. de; RESENDE, M.D.V.D de; OLIVEIRA, M.S.P. de; SANTOS, N.S.A.; CANUTO, E.L.; NOGUEIRA, O.L.; MULLER, A.A. Avaliação genética de progênies de polinização aberta de açaí (Euterpe oleracea) e estimativas de parâmetros genéticos. Cerne, Lavras, v.13, n.4, p. 376-383. 2007.

JARDIM, M.A.G. Aspectos da biologia reprodutiva de uma população natural de açaizeiro (Euterpe oleracea Mart.) no Estuário Amazônico. 1991. 90f. Dissertação (Mestrado em Agronomia) - Escola Superior de Agricultura Luiz de Queiroz, Piracicaba, 1991

OLIVEIRA, M.S.P de; CARVALHO, J.E.U de; NASCIMENTO, W.M.O do; MULLER, C.H. Cultivo do açaizeiro visando à produção de frutos. Belém-PA: Embrapa Amazônia Oriental, 2002. 18p (Circular Técnica, 026).

PACHECO, N.A.; BASTOS, T.X. Caracterização climática do Município de Tomé-Açu, PA. Belém-PA: Embrapa Amazônia Oriental, 2001. 18p. (Documentos, 87).

RESENDE, M.D.V.; DIAS, L.A.S. Aplicação da metodologia de modelos mistos (REML/BLUP) na estimação de parâmetros genéticos e predição de valores genéticos aditivos e genotípicos em espécies frutíferas. Revista Brasileira de Fruticultura, Jaboticabal, v.22, n.11, p.44-52, 2000.

RESENDE, M.D.V. de. Genética biométrica e estatística no melhoramento de plantas perenes, Brasília: Embrapa Informação Tecnológica, 2002. 975 p.

RESENDE, M.D.V.; BERTOLUCCI, F.L.G. Maximization of genetic gain with restriction on effective population size and inbreeding in Eucalyptus grandis. In: IUFRO CONFERENCE ON EUCALYPTUS PLANTATIONS: IMPROVING FIBRE YIELD AND QUALITY, 1995, Hobart, Australia. Proceedings papers... Sandy Bay: CRCTHF, 1995. p.167-170.

ROGEZ, H. Açaí: preparo, composição e melhoramento da conservação. Belém-PA: EDUFPA, 2000. 312p.

SOUZA, P.C.A. de. Aspectos ecológicos e genéticos de uma população natural de Euterpe oleracea Mart. no Estuário Amazônico. 2002. 60 f. Dissertação (Mestrado em Recursos Florestais) - Escola Superior de Agricultura Luiz de Queiroz, Universidade de São Paulo, Piracicaba, 2002.

STURION, J. A.; RESENDE, M.D.V. Eficiência do delineamento experimental e capacidade de teste no melhoramento genético da erva-mate (Ilex paraguariensis St. Hil.). Boletim de Pesquisa Florestal, Colombo, n.50, p.3-10. 2004.

TAIZ, L.; ZEIGER, E. Fisiologia vegetal. 3.ed. Porto Alegre: Artmed, 2004.719p. 